\title{
Measles outbreak investigation process in low- and middle-income countries: a systematic review of the methods and costs of contact tracing
}

\author{
Etheldreda L. Mbivnjo ${ }^{1,3}$ (D) Mary Lynch ${ }^{2} \cdot$ Jaci C. Huws ${ }^{3}$ \\ Received: 5 October 2020 / Accepted: 27 April 2021 / Published online: 15 May 2021 \\ (C) The Author(s), under exclusive licence to Springer-Verlag GmbH Germany, part of Springer Nature 2021
}

\begin{abstract}
Aim The occurrence of measles outbreaks has increased, and previously measles-free countries are experiencing a resurgence, making measles elimination by 2020 unlikely. Therefore, outbreak prevention and rapid response strategies will need to be intensified. This systematic review therefore examines whether contact tracing (CT) as compared to no CT is an effective means of reducing measles spread during outbreaks in low- and middle-income countries (LMICs).

Subject and methods A systematic review was conducted by searching six databases (CINAHL, Global Health, Medline, Cochrane Library, Web of Science and PubMed). The 17 included articles were appraised using the Critical Appraisal Skills Programme checklists and analysed using a narrative synthesis.

Results $\mathrm{CT}$ is often used alongside mass communication strategies and hospital record checks. Interviewing measles cases to identify contacts, and considering everyone who has shared a space with a case as a contact are common CT methods. Also, CT can be done backwards and/or forwards with the measles case as the focal point of the investigation process. The cost per case of an outbreak response dominated by CT is high especially in terms of labour for the health sector and productivity losses for households. However, overall outbreak expenditure can be low if CT results in fewer and less severe measles cases and a short outbreak duration.

Conclusion CT data as a standalone and comparative active surveillance approach in LMICs is scarce. If CT is initiated early, it can prevent large outbreaks, thereby reducing the economic burden of measles and drive LMICs towards measles elimination.
\end{abstract}

Keywords Measles outbreak $\cdot$ Contact tracing $\cdot$ Economic costs $\cdot$ Low- and middle-income countries

\section{Introduction}

Measles is a highly infectious airborne, acute and vaccinepreventable disease of viral origin (Moss, 2017). Prior to vaccine use and the revival of immunisation programmes, measles accounted for high child morbidity and mortality (Moss,

Etheldreda L. Mbivnjo

metheldreda@gmail.com

1 Department of Nursing, School of Health Sciences, Biaka University Institute of Buea, P.O. Box 77, Buea, Cameroon

2 Centre for Health Economics and Medicines Evaluation, School of Health Sciences, Bangor University, Gwynedd LL57 2EF, UK

3 School of Healthcare Sciences, Bangor University, Gwynedd LL57 2EF, UK
2017) with at least $95 \%$ of children aged under 15 years having had measles (World Health Organization [WHO], 2017), resulting in over 2 million deaths and 15,000 to 60,000 cases of blindness worldwide per annum. Safe and cost-effective combination (measles, rubella and mumps) vaccines have reduced global measles deaths to 535,000 in 2000 and 139,000 in 2010 (WHO, 2012).

The WHO set 2020 as the target for measles elimination in at least five of its regions and at the core of this global strategic plan for measles/rubella control and elimination, is outbreak preparedness and responsiveness (WHO, 2012). As an essential component of measles outbreak investigation and response (Ghebrehewet et al., 2016), contact tracing (CT) identifies, tracks and follows-up individuals who have had direct contact with an infected individual (WHO, 2015) to identify who infected the case and who the case may have infected (Sniadack et al., 2017). 
Numerous cases of measles go unreported (Strebel et al., 2011) and globally, approximately 20 million individuals are susceptible to measles and rubella (Measles and Rubella Initiative, 2018) while persistent endemicity of measles in some countries fosters importation and thus outbreaks in other countries (WHO, 2018). Though developed countries are not the main focus of this paper, such importations and poor vaccine coverage have been implicated in the re-emergence of measles in Europe and the United States of America (USA) over the past decade (Abad \& Safdar, 2015; Siani, 2019). In addition, outbreaks of measles are on the rise and there are indications that measles cases increased by $300 \%$ in the first quarter of 2019 with outbreaks occurring in 11 low- and middleincome countries (LMICs) relative to the same period in 2018 (WHO, 2019). Consequently, eliminating measles by the year 2020 (WHO, 2012) has been considered an unrealistic ambition given that none of the six WHO regions had achieved an earlier 2015 milestone of $95 \%$ coverage in supplementary vaccination in every health district (Dabbagh et al., 2018; Orenstein et al., 2018). Furthermore, there are indications that the core elements of the global strategic plan for measles are executed partially or are not tailored to local needs (Orenstein et al., 2018), and 13 countries are significantly off-track for measles elimination in the WHO African Region (WHO Regional Committee for Africa, 2017).

CT is resource-intensive and its use in outbreaks may be limited by the competition for scarce resources with other outbreak response activities. Delaying to initiate $\mathrm{CT}$ because of limited resources makes a later attempt to trace contacts more expensive as exposures would have increased exponentially (WHO, 2015). LMICs experience the highest measles incidence and mortality with very few certified as measles-free in 2017 (Dabbagh et al., 2018); however, the share of healthcare in their government budgets remains low with an overreliance on donors and the private sector (PiattiFünfkirchen et al., 2018). Also, donor fatigue and reduced interest of countries in issues perceived as mainly associated with developing countries have been identified (Hinman, 2018). This represents a potential challenge in obtaining financial support for measles outbreak response. Therefore, gaining insights into the economic costs of $\mathrm{CT}$ is essential as it will build a case for a full economic evaluation and inform investment in preventive strategies to sustain measles eradication efforts as well as those of other infectious diseases. The aim of this systematic review is to investigate whether $\mathrm{CT}$ as compared to no $\mathrm{CT}$ is an effective means of reducing the spread of measles during outbreaks in LMICs by determining methods used in CT and the economic costs of $\mathrm{CT}$ during measles outbreaks.

\section{Methods}

This study used a systematic review approach as outlined in the Preferred Reporting Items for Systematic Reviews and Meta-Analyses (PRISMA) guidelines (Moher, Liberati, Tetzlaff, Altman, and Group, 2009), whose completed checklist can be found in Online Resource 1. The inclusion criteria for this study were as follows: journal articles published in English, within the past 30 years, of any study design and that reported measles as an outbreak; use of $\mathrm{CT}$ in measles outbreak investigations; and measles outbreaks within the community and/or hospital. The exclusion criteria were: journal articles reporting rubella only or isolated measles cases (where no outbreak was declared), or measles outbreaks in developed countries even if reported to have been imported from a LMIC; and studies published in a language other than English, or for which the full text was not available.

\section{Search strategy and study selection}

Six electronic databases (CINAHL, Global Health, Medline, Cochrane Library, Web of Science and PubMed Central Open Access) were searched in July 2019. The selection of search terms was guided by the population, intervention, comparator, outcome (PICO) format as shown in Table 1 below and the use of closely related words from background/existing literature. These terms were applied individually then combined using two Boolean operators "OR" and "AND" as outlined in Table 2 below. Other relevant articles were identified by hand searching the reference lists of included articles. Two main reviewers (ELM and ML) decided on the search terms to be used and the data to be extracted. Any disagreements regarding literature inclusion, data extraction or quality assessment were reviewed and settled by the third reviewer $(\mathrm{JCH})$.

\section{Quality appraisal}

The various Critical Appraisal Skills Programme (CASP) checklists were used to appraise the quality of evidence in the selected articles (CASP, 2018). The Strengthening the Reporting of Observational Studies in Epidemiology (STROBE) checklist (von Elm et al., 2007) was applied to critically appraise selected articles that had a cross-sectional design since a checklist dedicated to cross-sectional studies was not available within the CASP at the time of this review.

\section{Data extraction}

A form for data extraction was created on Excel, piloted and refined to improve its validity. ELM and ML independently reviewed the included studies for data on article features (author, title, country of origin and design), participant 
Table 1 Keywords used in database searches

\begin{tabular}{ll}
\hline $\begin{array}{l}\text { PICO } \\
\text { framework }\end{array}$ & Keywords \\
\hline Population & $\begin{array}{r}\text { low- and middle-income countries, developing countries, under developed countries, low } \\
\text { resource countries, third world countries, global south, heavily indebted poor countries and } \\
\text { least developed countries }\end{array}$ \\
Intervention & $\begin{array}{c}\text { Contact tracing, case finding, case investigation, case search, rumour surveillance, contact } \\
\text { management, contact investigation and transmission chain tracking }\end{array}$ \\
Comparator & $\begin{array}{l}\text { No contact tracing } \\
\text { Measles outbreak control, measles outbreak termination, measles outbreak interruption, } \\
\text { measles epidemic control, reduce measles spread and decrease measles transmission }\end{array}$ \\
\hline
\end{tabular}

demographics, methods, results, limitations and funding. The Mendeley referencing software was used to track all references and selected full-text articles.

\section{Data analysis and synthesis}

An overview of the included studies was first given using a descriptive synthesis. This was followed by a thematic analysis to evaluate the exhaustiveness of evidence within the included articles and to determine the relationships that exist within and between them (Centre for Reviews and Dissemination University of York, 2009). A narrative synthesis of the findings of included studies was conducted together with an explanation of how and for whom CT works. Also, the relationship between and within these articles was explored by concept mapping and a visual illustration of the relationship between study features/results. Lastly, a critical reflection on the synthesis process was conducted to identify its strengths and weaknesses and to inform the review's conclusions and recommendations.

\section{Study registration}

This systematic review was internally registered with the Academic Ethics Committee for Bangor University and was granted exemption from requiring ethics approval (as the study did not involve human participants), and externally with PROSPERO (an international Prospective Register of Systematic Reviews), registration number CRD42019142794.

\section{Results}

\section{The article selection process}

Database searches identified 617 studies and the details of this process has been presented in Table 2 above. After screening study titles and abstracts, 371 and 113 articles were excluded respectively because the research was not conducted in a LMIC, or the area of focus was not measles or measles outbreak, or there was no CT or outbreak investigation. The full-texts of 133 articles were read for eligibility and 109 articles were excluded because the articles did not have content on CT or the full-text was not in English. Across all databases, 11 duplicates were removed leaving 13 articles whose reference lists were hand-searched revealing 4 additional articles and resulting in a total of 17 articles included in this review. Figure 1 below is a modified PRISMA flow diagram (Moher et al., 2009) showing how the study selection was conducted. A summary of the article selection process can be found in Online Resource 2. Also see Online Resource 3 for the study selection process of each database.

\section{Characteristics of selected studies}

The following subsections have incorporated the PICO framework (though the comparator [C] is absent as studies comparing CT to no CT were not found) into the description of the main study characteristics and more information can be found in Tables 3 and 4 below.

\section{Study population}

From the 17 studies included in this review, four articles were from China (Jin et al., 2011; Ma et al., 2016; Ma et al., 2017; Zhang et al., 2015), three from Senegal (Cisse et al., 1999; Garenne \& Aaby, 1990; Whittle et al., 1999), two from the Federated States of Micronesia (Hales et al., 2016; Pike et al., 2017), and one each from India (Rathi et al., 2017), Bolivia (Quiroga et al., 2003), Brazil (Lemos \& Franco, 2017), Venezuela (Sarmiento et al., 2011), Republic of Marshall Islands (Marin et al., 2006), Romania (Njau et al., 2019), Tanzania(Goodson et al., 2010), and Ethiopia (Wallace et al., 2014). Eleven of the studies involved both male and female participants and the remaining six had no data on sex distribution of subjects (Garenne \& Aaby, 1990; Marin et al., 2006; Pike et al., 2017; Quiroga et al., 2003; Sarmiento et al., 2011; Wallace et al., 2014). Most (88.2\%) of the studies included both children and adults, but two studies included children (Whittle et al., 1999) and young adults (Jin et al., 2011) 


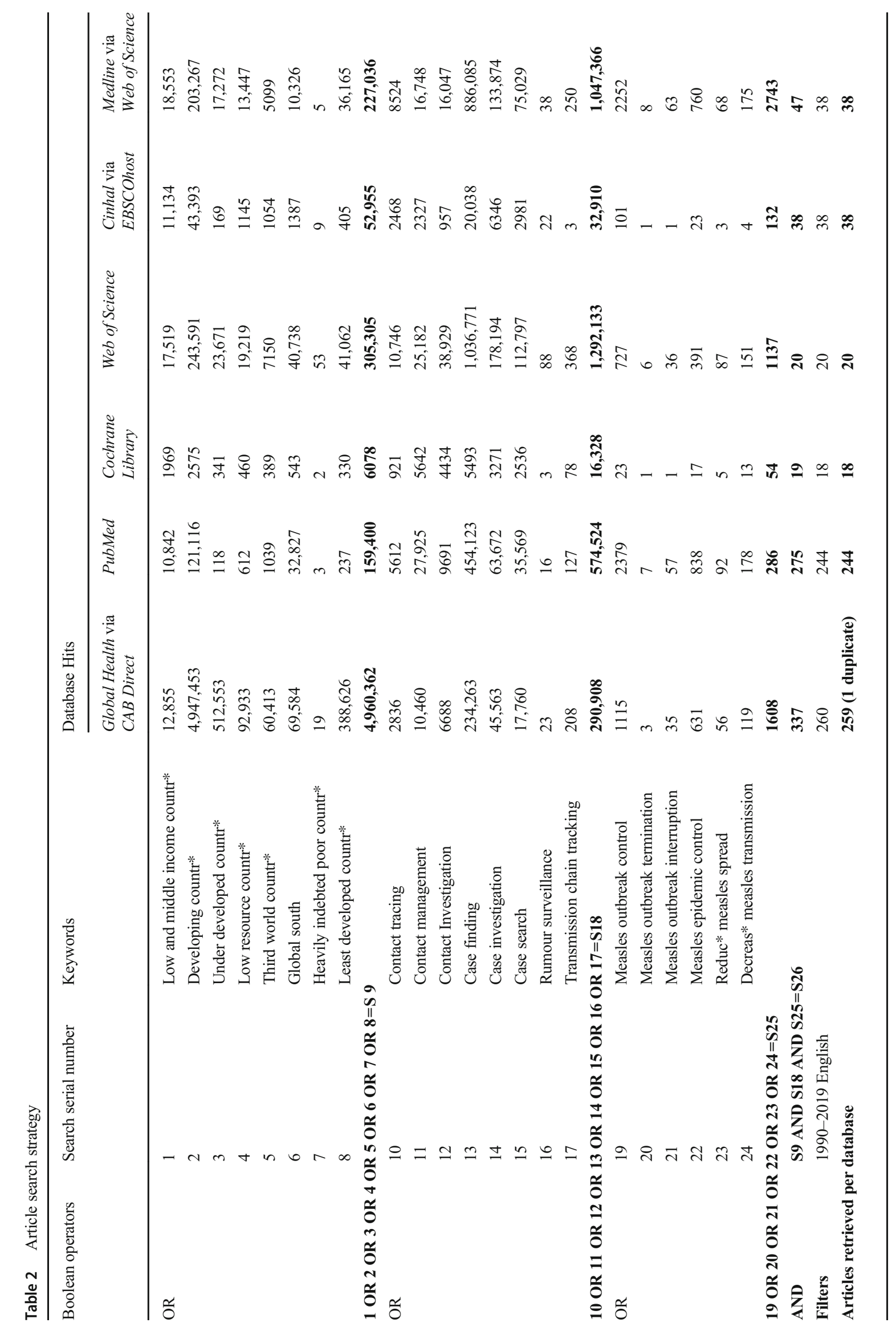


Fig. 1 Article selection process
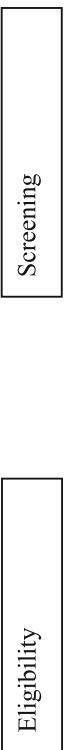

only. With regard to population density, three studies reported high numbers of persons per household (Garenne \& Aaby, 1990; Hales et al., 2016; Marin et al., 2006). Several studies highlighted crowding at public events such as football competitions (Lemos \& Franco, 2017), university environments (Jin et al., 2011; Rathi et al., 2017), hospital units (Zhang et al., 2015), large cities (Goodson et al., 2010; Quiroga et al., 2003) and work environments (Ma et al., 2017).

\section{Interventions}

To trace contacts, cases were located through surveillance records and contacts solicited through case or caregiver/
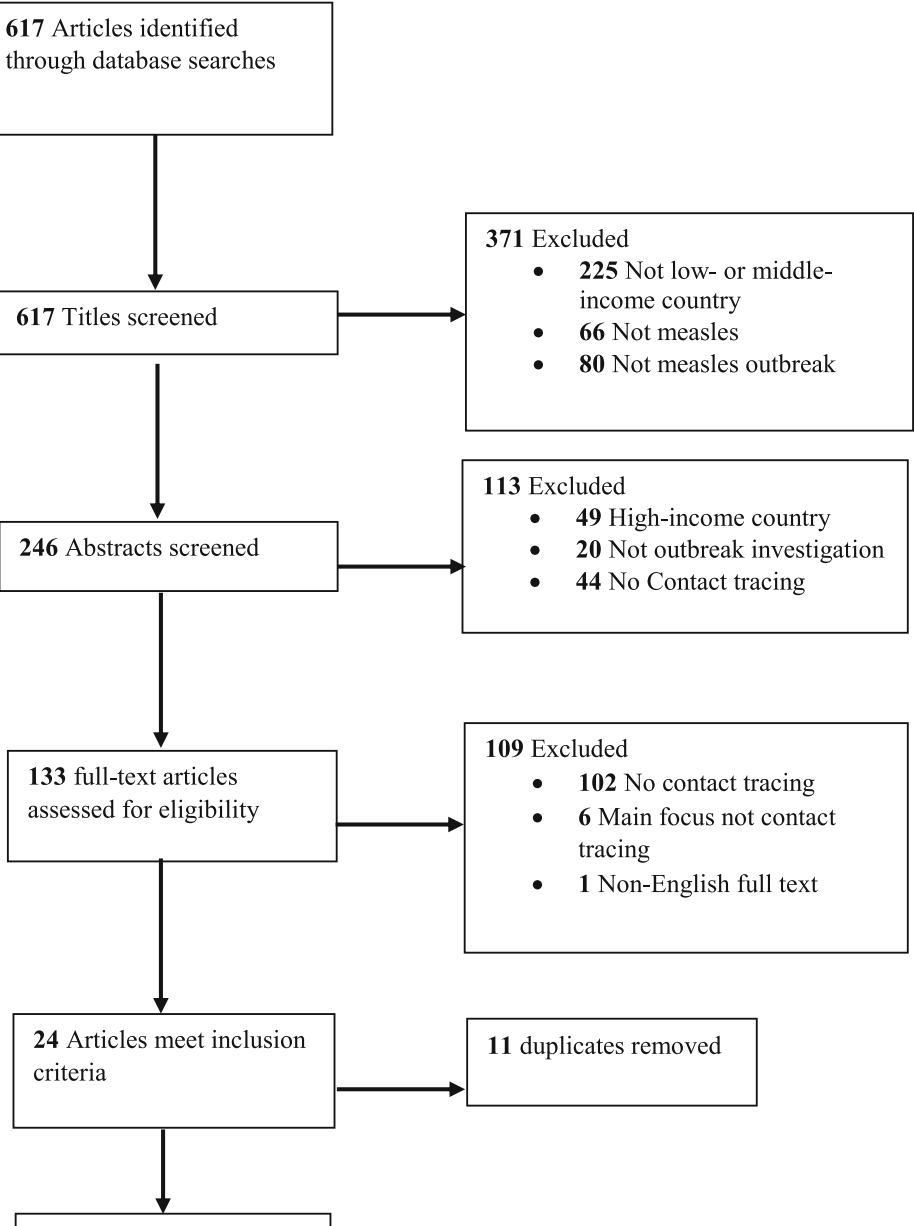

13 Articles retained

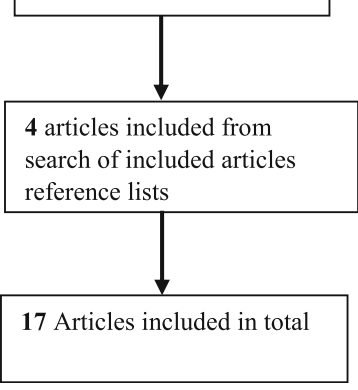

parent interview (Garenne \& Aaby, 1990; Goodson et al., 2010; Hales et al., 2016; Ma et al., 2016; Whittle et al., 1999). In other studies (Jin et al., 2011; Ma et al., 2017; Marin et al., 2006; Quiroga et al., 2003; Rathi et al., 2017; Zhang et al., 2015), the contacts were approached directly after locating the places where the cases worked or lived. One study established a contact list and followed-up everyone sharing a home or enclosed space with the case (Sarmiento et al., 2011), another obtained a list of exposed pupils from the school register (Cisse et al., 1999), and a third used the picture of a measles case to search for contacts within the community (Lemos \& Franco, 2017). CT was implemented alongside mass communication strategies (Lemos \& Franco, 2017; Ma 


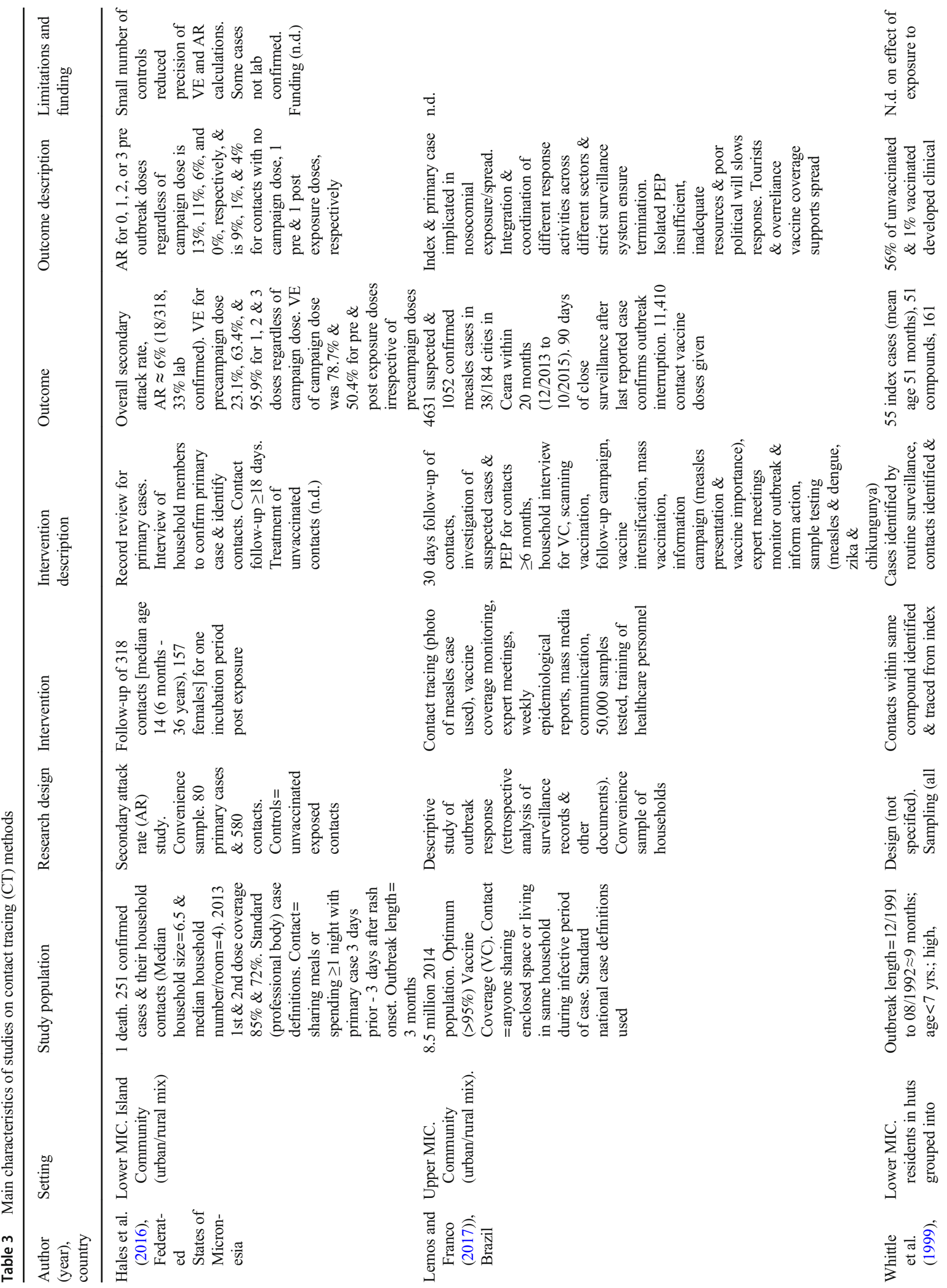




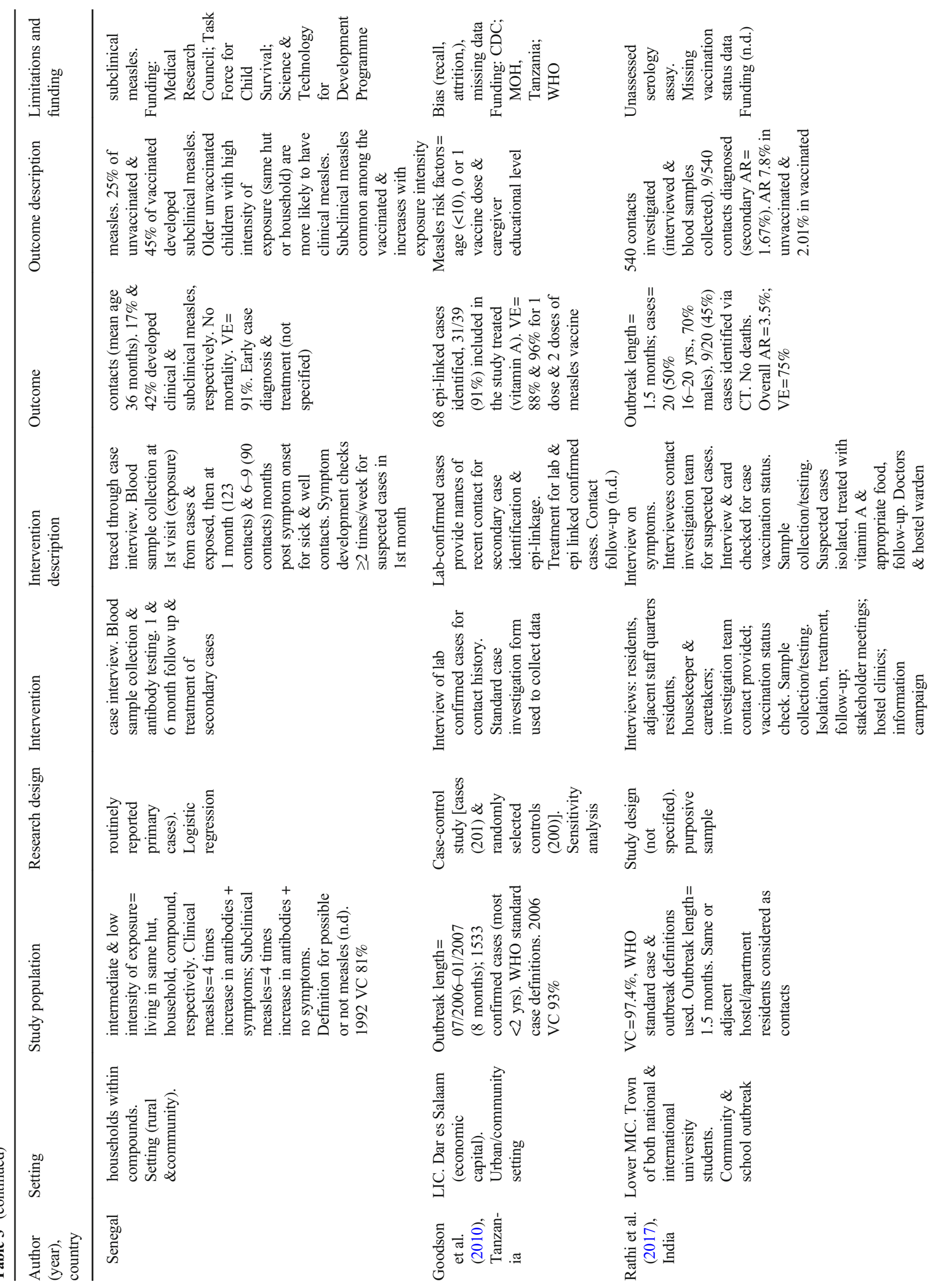




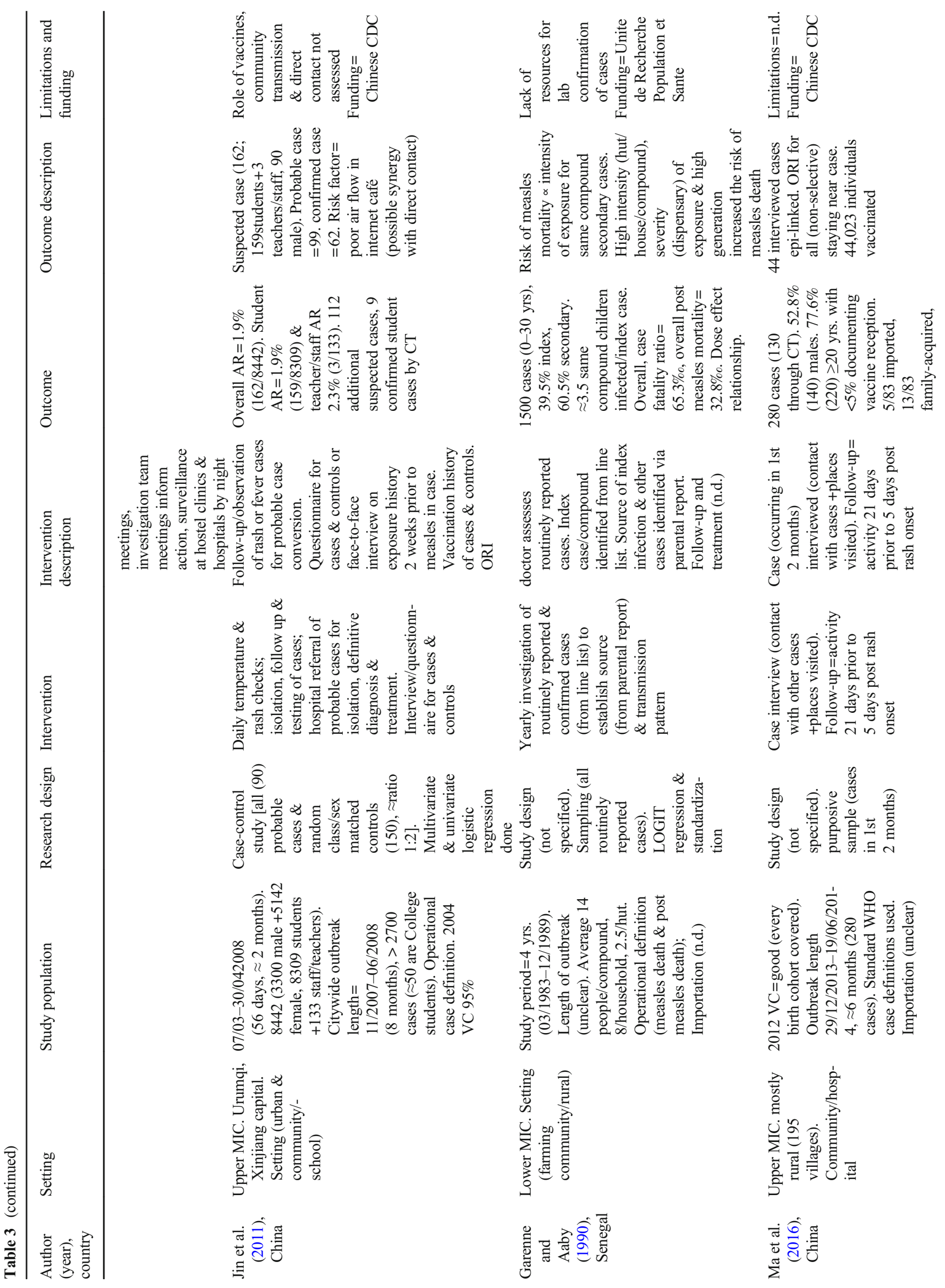




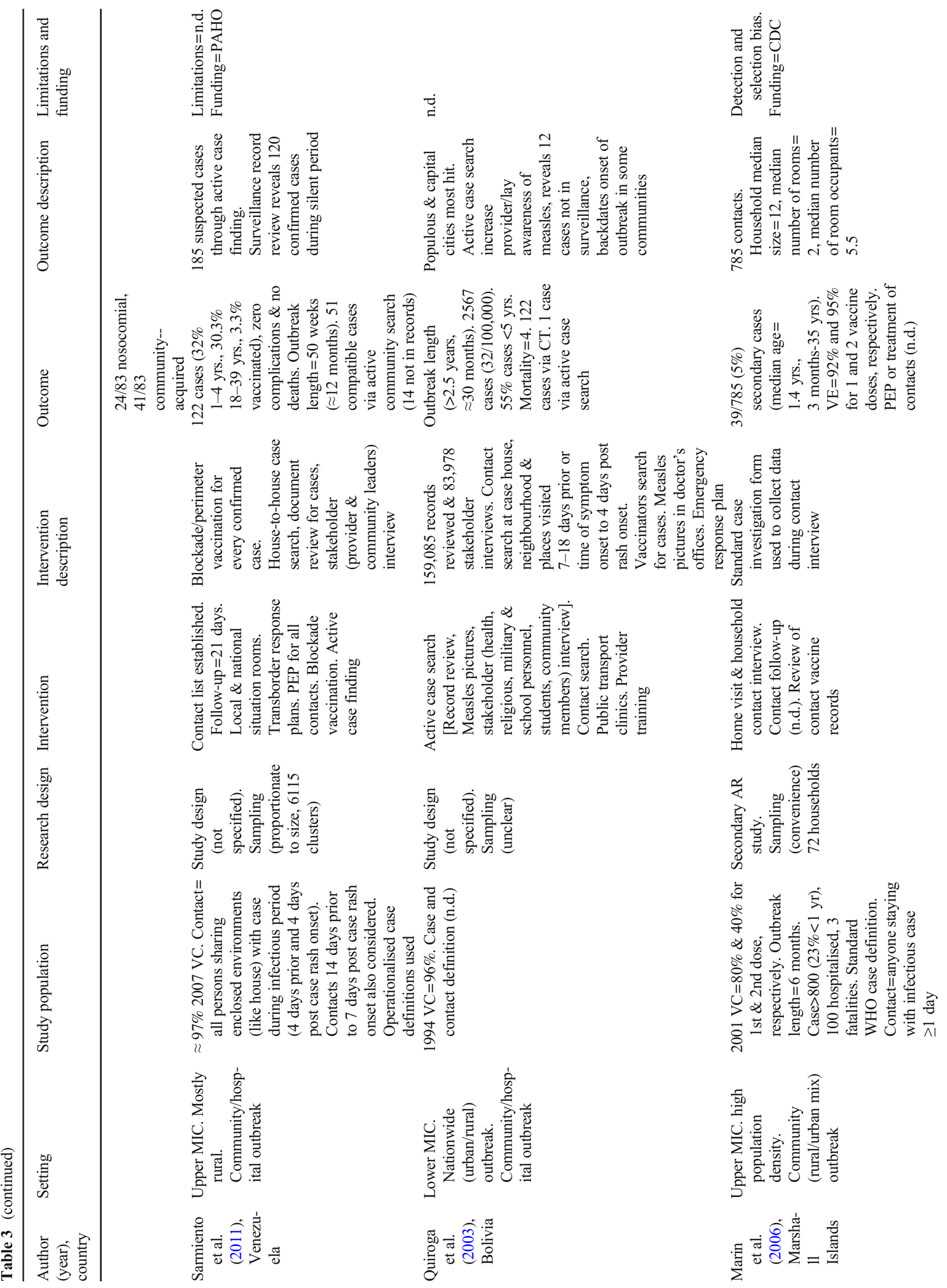




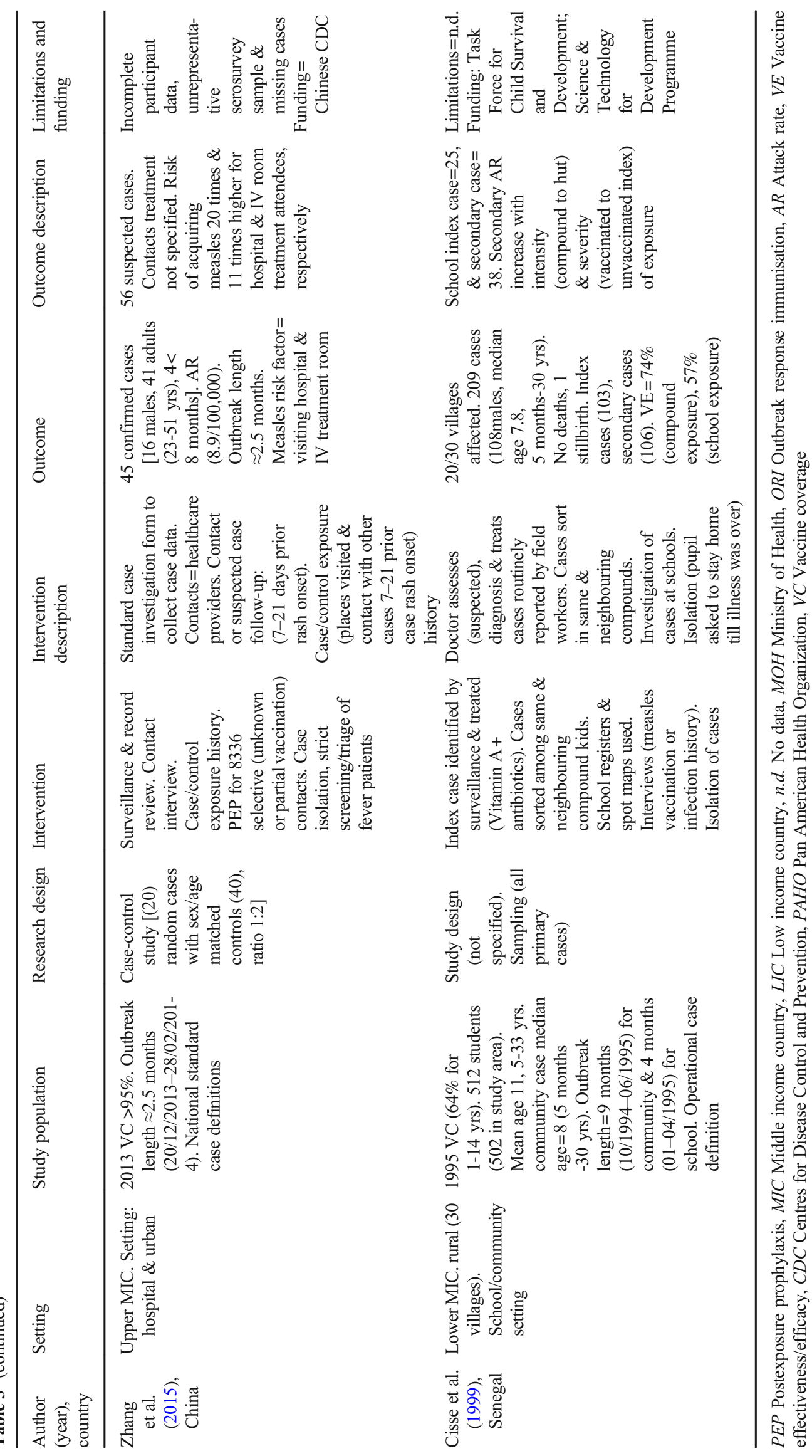




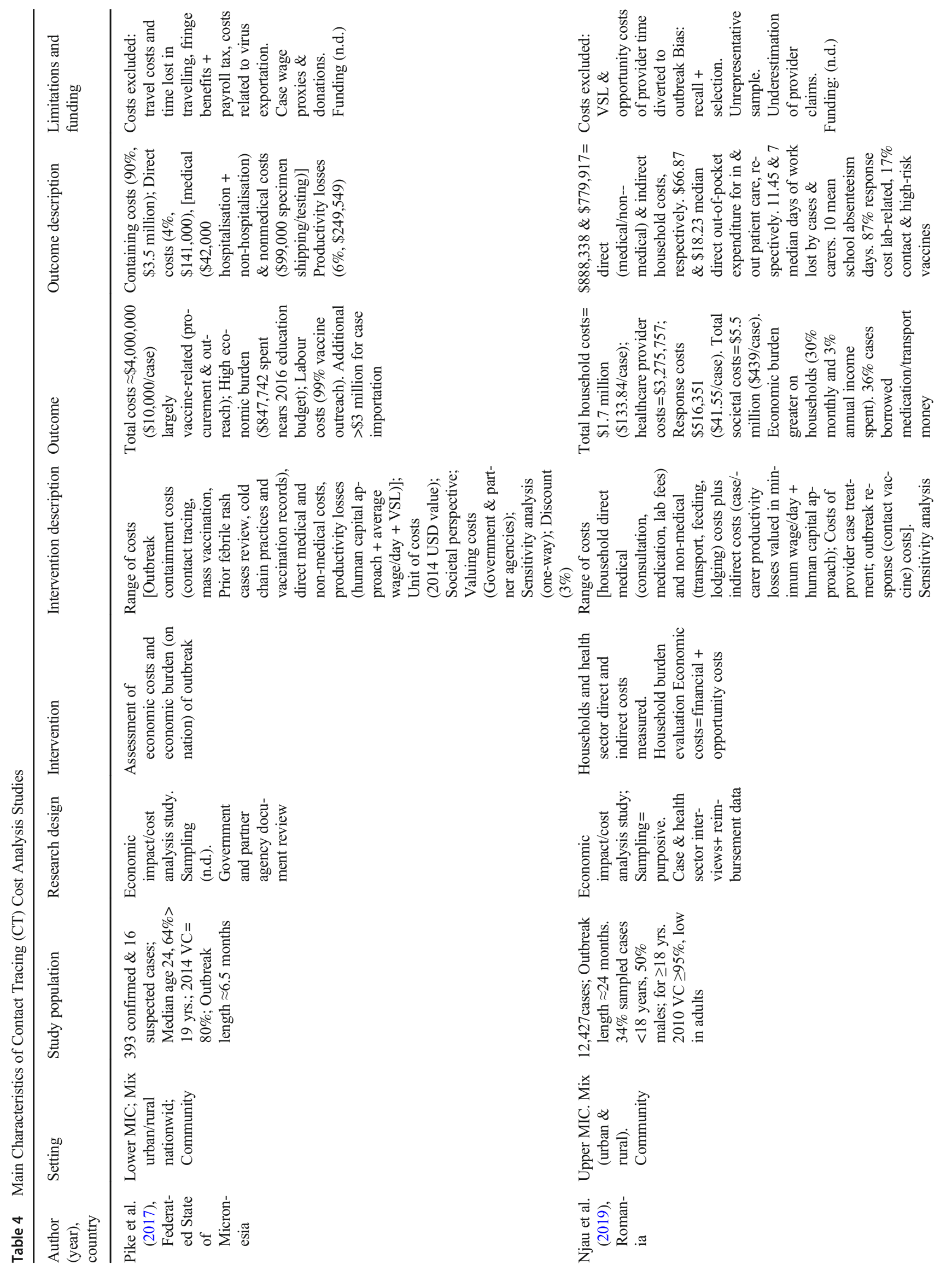




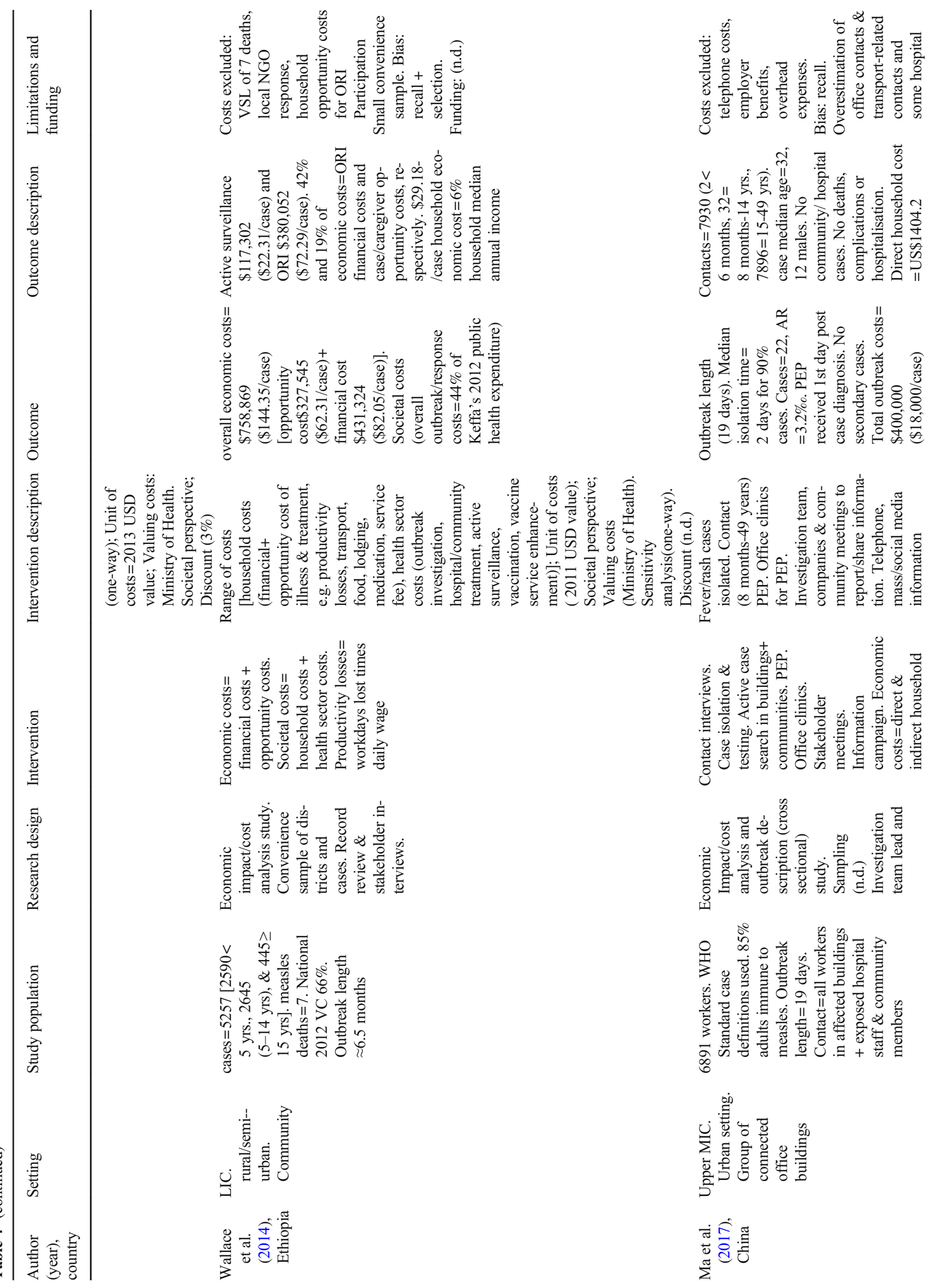




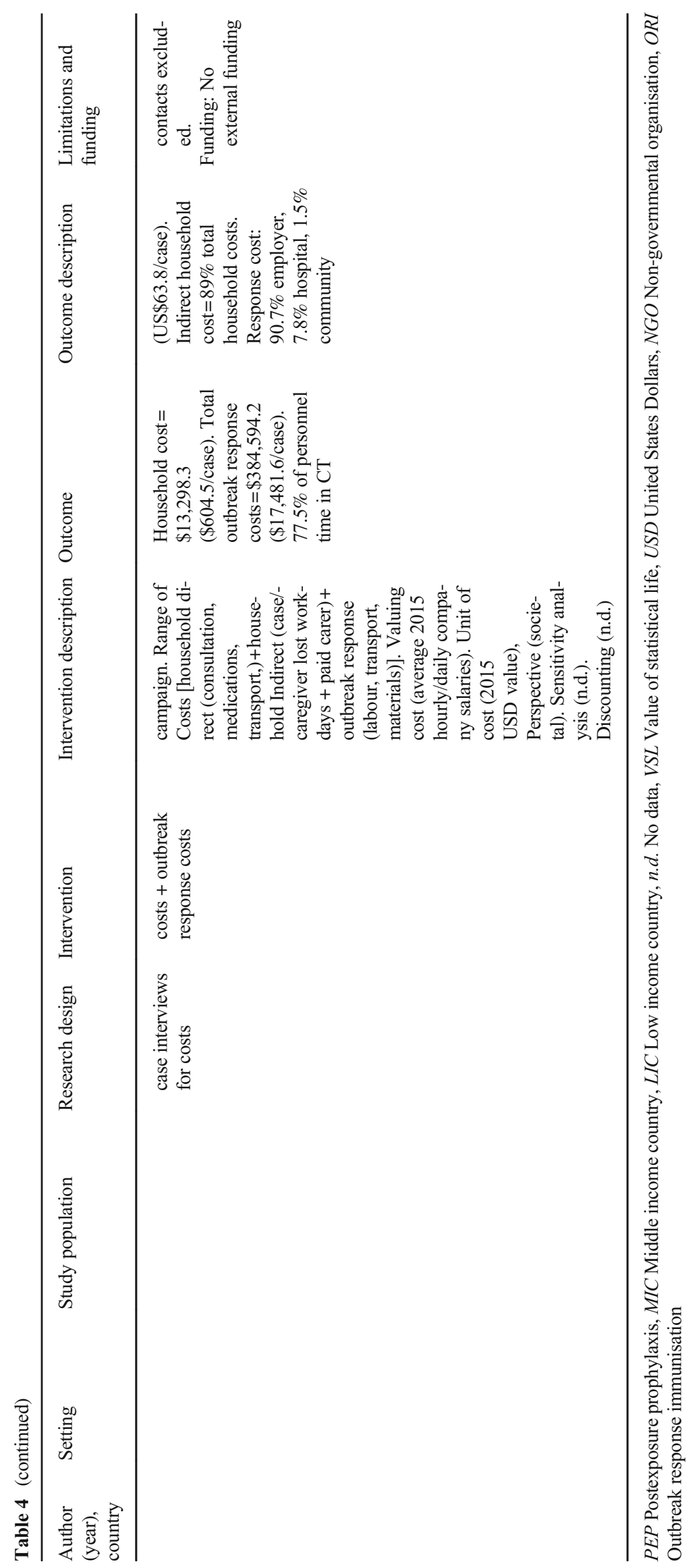


et al., 2017; Rathi et al., 2017), and vaccinators to search for cases (Quiroga et al., 2003).

All the cost analysis studies adopted a societal perspective, used the United States Dollars (USD) as the unit of measuring costs, and collected data on both direct and indirect costs of the outbreak and response. One study costed CT among other outbreak response activities (Pike et al., 2017) and contact vaccination was part of the outbreak response cost items in another study (Njau et al., 2019). In an Ethiopian study (Wallace et al., 2014), active surveillance was part of the health sector costs, and a Chinese study estimated the proportion of personnel time used in $\mathrm{CT}$ as part of outbreak response activities (Ma et al., 2017).

\section{Outcomes}

Contact tracing and prevention of measles spread: In this systematic review, prevention of measles spread was assessed by length of outbreak and magnitude/size of outbreak (number of cases and deaths) which is similar to the case-day index, a measure validated by comparing contacts per case and contacts per day ratios (Ortega-Sanchez et al., 2014).

The length of the measles outbreak in the included studies varied from 19 days (Ma et al., 2017) to over 30 months (Quiroga et al., 2003). Six of the studies had outbreaks lasting for 6 months or less (Hales et al., 2016; Jin et al., 2011; Ma et al., 2016; Marin et al., 2006; Rathi et al., 2017; Zhang et al., 2015). One study's outbreak duration was 8 months (Goodson et al., 2010), two other studies had outbreaks for 9 months (Cisse et al., 1999; Whittle et al., 1999), another for 12 months (Sarmiento et al., 2011), and one other for as long as 20 months (Lemos \& Franco, 2017). One study (Garenne \& Aaby, 1990) had an unclear outbreak length as the study seems to have involved several outbreaks over a 4-year-period.

The number of cases identified in some studies were 1500 cases (Garenne \& Aaby, 1990), 280 cases (Ma et al., 2016), 122 cases (Sarmiento et al., 2011), 2567 cases (Quiroga et al., 2003), 45 cases (Zhang et al., 2015), and 209 cases (Cisse et al., 1999). No deaths were recorded by some studies (Ma et al., 2017; Rathi et al., 2017; Sarmiento et al., 2011; Whittle et al., 1999). A small proportion of studies reported few deaths: four deaths (Quiroga et al., 2003), one stillbirth (Cisse et al., 1999), one death (Hales et al., 2016) and a case fatality ratio of $6.5 \%$ (Garenne \& Aaby, 1990).

Economic costs of contact tracing: It is recognised that there is an economic cost associated with CT. Containment cost (contact tracing inclusive), productivity losses, and direct medical and non-medical cost accounted for $90 \%, 6 \%$ and $4 \%$ of the outbreak expenditure, respectively (Pike et al., 2017). Research findings indicate that $17 \%$ of total outbreak cost was spent on contact and high-risk group vaccines ( $\mathrm{Njau}$ et al., 2019). The economic cost of active surveillance was estimated at $\$ 117,302$ (\$22.31/case) compared to $\$ 380,052$ (\$72.29/case) for outbreak response immunisation (Wallace et al., 2014) with $77.5 \%$ of personnel time spent on CT alone (Ma et al., 2017).

\section{Quality and risk of bias assessment}

Two secondary attack rate studies (Hales et al., 2016; Marin et al., 2006) and four studies whose design was not explicitly stated (Cisse et al., 1999; Garenne \& Aaby, 1990; Rathi et al., 2017; Whittle et al., 1999) were classified as cohort studies based on the reviewer's evaluation and the corresponding CASP tool (CASP, 2018) was used to appraise their quality (see Table 1, Online Resource 4). All the studies had clearly stated objectives, the method of recruiting cohorts was acceptable and the findings were in line with existing evidence with huge practice implications.

Three of the included studies used a case-control design (Goodson et al., 2010; Jin et al., 2011; Zhang et al., 2015) and were assessed for quality using the CASP case-control tool (CASP, 2018). In these three studies, all cases and controls were randomly selected and treated equally, confounding was minimised, the treatment effects were large, and each study's findings were in line with existing evidence (see Table 2, Online Resource 4).

One of the included studies was clearly identified as descriptive (Lemos \& Franco, 2017) and three others whose design was not clearly stated (Ma et al., 2016; Quiroga et al., 2003; Sarmiento et al., 2011) were added to this category because there was no investigation of causal relationships (Aggarwal \& Ranganathan, 2019) and assessed using the STROBE checklist (von Elm et al., 2007). These studies were scientifically acceptable; clearly and elaborately described measles outbreaks plus response activities (see Table 3, Online Resource 4).

Four of the studies (Ma et al., 2017; Njau et al., 2019; Pike et al., 2017; Wallace et al., 2014) were partial economic evaluations. To assess their quality, the CASP tool for full economic evaluations was adapted by excluding its comparator and marginal analysis items and including items common to Consolidated Health Economic Evaluation Reporting Standards (CHEERS) (Husereau et al., 2013) and Quality of Health Economic Studies (QHES) (Ofman et al., 2003) tools whose assessment findings have been shown to be comparable (Monten et al., 2017). All four studies had clear objectives; discussed how costs were identified, valued and measured; and clearly stated the perspective of economic evaluation (see Table 4, Online Resource 4).

For risk of bias assessment, non-random sampling methods such as convenience sampling (Hales et al., 2016; Lemos \& Franco, 2017; Marin et al., 2006; Zhang et al., 2015) or purposive sampling (Ma et al., 2016; Rathi et al., 2017) and nonresponse bias (Goodson et al., 2010; Ma et al., 2016; Wallace 
et al., 2014; Whittle et al., 1999) were the main reasons for selection bias. For information bias ratings, most of the studies were found to have recall bias (Cisse et al., 1999; Garenne \& Aaby, 1990; Goodson et al., 2010; Jin et al., 2011; Marin et al., 2006; Rathi et al., 2017; Sarmiento et al., 2011; Wallace et al., 2014; Zhang et al., 2015) and a few others detection bias (Garenne \& Aaby, 1990; Lemos \& Franco, 2017; Marin et al., 2006; Sarmiento et al., 2011). For confounding, the reasons were variable across studies and details can be found in Online Resource 5.

\section{Thematic analysis and narrative synthesis}

\section{Methods of contact tracing}

CT can be done by case/caregiver referral where cases are located through surveillance records and contacts are solicited by interviewing the case or caregiver/parent (Garenne \& Aaby, 1990; Goodson et al., 2010; Hales et al., 2016; Ma et al., 2016; Whittle et al., 1999). CT can also be conducted by shared space identification with contacts approached directly after locating the places where the cases work or live through routinely reported data (Jin et al., 2011; Ma et al., 2017; Marin et al., 2006; Quiroga et al., 2003; Rathi et al., 2017; Zhang et al., 2015) or by establishing a contact list and following up everyone sharing a home or enclosed space with the case (Sarmiento et al., 2011).

School registers can also be used as a method of CT by obtaining a list of exposed pupils and mapping out classroom sitting positions with the help of teachers (Cisse et al., 1999). Other school-related outbreaks utilise teachers for daily rash and temperature/fever checks (Jin et al., 2011) or interview staff working at student residential halls to report new cases (Rathi et al., 2017). The picture of a measles case can also be used to search for contacts within the community (Lemos \& Franco, 2017).

CT is part of an integrated active surveillance approach. Therefore, it is often combined with other strategies such as mass communication strategies (Lemos \& Franco, 2017; Ma et al., 2017; Rathi et al., 2017), hospital record checks (Lemos \& Franco, 2017; Sarmiento et al., 2011; Zhang et al., 2015) or having vaccinators search for cases as they move from house to house (Quiroga et al., 2003).

\section{Forward versus backward contact tracing}

With the index case as the focal point, CT can be achieved via forward and/or backward modes. The infective or transmissibility period of the case can be used to determine who the case might have infected ('to whom'), which helps in the identification of secondary cases and the construction of case generations. During home visits, information about how measles was contracted can be obtained (Whittle et al., 1999) for the classification of secondary cases if rash onset occurred 7 to 21 days post rash onset in the primary case (Hales et al., 2016).

To establish the source ('from whom') of the infection, identify additional cases and understand transmission patterns, travel history of the case during the incubation period is collected. Cases are interviewed about hospitals visited during the 7 to 21 days prior to measles rash onset (Zhang et al., 2015), contacts are monitored daily for symptom development up to 21 days post the last date of possible exposure (Ma et al., 2017) and suspected cases are asked for any travel in the 21 days prior to rash onset (Lemos \& Franco, 2017).

Combined approaches involve obtaining the history of activities from cases 21 days prior to rash onset up to 5 days post rash onset (Ma et al., 2016) and collecting information of all places visited by cases in 7-18 days before rash onset or during the period between the beginning of respiratory symptoms until 4 days after rash onset (Quiroga et al., 2003). In addition, simply collecting information on recent contacts without stating any timelines has been used (Goodson et al., 2010).

\section{Contact tracing methods and case identification}

Evidence would indicate that there is a link between shared space identification as a method of CT and identification of additional cases through CT. Four (Jin et al., 2011; Quiroga et al., 2003; Rathi et al., 2017; Sarmiento et al., 2011) out of the six studies that reported cases obtained through CT had considered everyone sharing an enclosed space with the case as a contact. One study (Sarmiento et al., 2011) reported 14 cases that were absent from surveillance records and uncovered 120 confirmed cases during a period which was initially thought to be epidemiologically silent. In addition, another study (Quiroga et al., 2003) identified 12 cases not present in surveillance records, the third study (Rathi et al., 2017) identified 9 additional cases which accounted for $45 \%$ of all cases in the outbreak, and the fourth (Jin et al., 2011) identified 112 additional suspected cases of which 9 were confirmed. Thus, shared space identification appears to be a more comprehensive CT strategy.

\section{Economic costs of contact tracing}

None of the included studies determined the economic costs of CT separately from other outbreak activities but CT was incorporated into the costing of the entire outbreak. Consequently, costs will be analysed as per the study finding with highlights on $\mathrm{CT}$ when available. In addition, due to the perspectives and unit of costs being similar, it is possible to make comparisons where possible across the studies.

Contact tracing cost and outbreak magnitude: The total outbreak cost ranged from $\$ 0.4$ million (Ma et al., 2017) to $\$ 5.5$ 
million (Njau et al., 2019) and the cost per case from $\$ 144.35$ (Wallace et al., 2014) to $\$ 18,000$ (Ma et al., 2017) among the studies included in this review. In one study (Ma et al., 2017) where the cost/case was the highest, overall outbreak costs were lowest, with the lowest number (22 confirmed) of cases and shortest outbreak length (19 days) when compared to two other studies each having 6.5 months outbreak length with 409 cases (Pike et al., 2017) and 5257 cases (Wallace et al., 2014), respectively, or with another of 24 months outbreak duration and 12,427 cases (Njau et al., 2019). In this same study (Ma et al., 2017), CT was used extensively and expended $77.5 \%$ of provider time as part of outbreak response cost that formed the bulk of the total outbreak expenditure. This indicates that timely and robust CT may be effective (fewer cases, less severity of cases, no/less deaths, shorter outbreak) and could be good value for money.

The overall outbreak cost is a function of the comprehensiveness of the range of costs considered in cost analysis and the extent to which each response intervention was used. In one study (Wallace et al., 2014), seven measles deaths were not factored into the calculation of productivity losses and had an overall outbreak cost of approximately 0.8 million, which is just above the average cost estimate $(\approx 0.6$ million, without application of income elasticity) for the lone death in another study (Pike et al., 2017) with approximately 4 million total outbreak cost. Given that these two studies have similar outbreak lengths ( 6.5 months), the huge disparity in their overall outbreak costs could partly be as a result of differential cost inputs. Also, while the latter (Pike et al., 2017) specifically stated CT as part of outbreak response, the former (Wallace et al., 2014) used the umbrella term 'active surveillance' which could be indicative of minimal CT, and thus the reason for the variance in cost. Also, the extensive use of CT in another study (Ma et al., 2017) may explain its high $(\$ 18,000)$ cost per case.

Direct versus indirect contact tracing costs: For the health sector, direct labour costs tend to be the main driver of outbreak response costs. In the Romanian study (Njau et al., 2019), provider reimbursement for the treatment/ management of cases was $\$ 3.3$ million which is more than half of the 5.5 million spent in total for outbreak response. In the Micronesian study, labour cost constituted the main economic burden for the country (Pike et al., 2017). Again, 98.4\% of outbreak control costs were from labour in a Chinese study (Ma et al., 2017).

For households, the indirect costs of workdays lost because of measles infection/death (either as a case or caregiver) dominated expenditure. For example, the opportunity costs of lost workdays accounted for $89.4 \%$ of the total household costs in one Chinese study (Ma et al., 2017). Similarly, $87 \%$ of household costs have been shown to be opportunity costs (Wallace et al., 2014).

\section{Discussion}

The aim of this systematic review was to identify methods used in $\mathrm{CT}$ and the associated economic costs. The 17 studies included in this review had varied research designs, making it impossible to conduct any form of meta-analysis. None of these studies compared CT to no CT; thus, no empirical data addressing the review question was found and it was not possible to determine the effectiveness of $\mathrm{CT}$ as compared to no $\mathrm{CT}$ in preventing measles spread during outbreaks in LMICs. Also, none of the included studies assessed CT or its costs separately. Disaggregation of data on CT and the paucity of studies on resources used in $\mathrm{CT}$ and associated costs has been previously identified (Canadian Agency for Drugs and Technologies in Health, 2015) and could be explained by the insufficiency of CT when infection spreads rapidly and contacts increase exponentially (Dhillon \& Srikrishna, 2018).

\section{Methods of contact tracing}

The evidence would suggest that shared space identification was the most frequent method of CT and is associated with finding additional cases. A similar approach in CT is where contacts were located by identifying homes of hospitalised index cases (Mupere et al., 2006). Similar findings indicate that CT helps in identifying exposed co-workers and patients (Jones et al., 2015) as well as exposed individuals who have boarded the same plane as the imported case (Beard et al., 2011).

Asking cases/caregivers to recall contacts was also common among the selected studies. This same approach was employed in the economic assessment of hospital-associated measles outbreaks in the USA, where suspected measles cases were interviewed for a list of contacts (Chen et al., 2011). This method is subject to bias as contacts unknown to the cases may continue to spread infection - if infected (Dhillon \& Srikrishna, 2018), and may not be investigated, thus limiting study findings (Ching et al., 2016).

With the index case as the focal point, CT can be done backward and/or forward to determine the source of the infection and onward spread by using the case's exposure and infective periods, respectively. In the UK, the health protection teams use both perspectives by obtaining information on contact/travel history and close contacts (Smith, 2018). Consequently, unprotected contacts have been advised to vaccinate or self-exclude for 21 days (the incubation period) and suspected cases isolated for 4 days post rash onset (Begum et al., 2017), which corresponds to the infective period. Therefore, CT can be viewed as a continuum of 'from whom' (backward) 'to whom' (forward) and CT methods can be applied in either (forward or backward) or both directions. 


\section{Costs of contact tracing}

Evidence from the included studies show that the cost per case of CT is high. The costs of investigating possible, probable and unreported cases has accounted for $67 \%$ of total outbreak costs with direct public health costs (CT inclusive), almost 1.5 times that of hospital admissions (Ghebrehewet et al., 2016). Provider labour costs/time is the main driver of high CT costs within the included studies. This is not an isolated observation as staff absenteeism owing to lack of immunisation evidence, exposure or measles accounted for $56 \%$ of total outbreak costs during a hospital-associated outbreak (Chen et al., 2011). From the provider perspective, labour cost estimates of a single imported case of measles can range from $\$ 264$ to $\$ 300$ per contact (Coleman et al., 2012). Also, an estimation of the health sector economic burden of 16 measles outbreaks in the USA (Ortega-Sanchez et al., 2014) was at 42,635 to 83,133 personnel hours spent in tracing 8936 to 17,450 contacts with an equivalence of annual full-time hours of 20 to 39 providers spent on investigating these measles outbreaks.

Evidence would suggest that if CT is initiated early (Ma et al., 2017), secondary cases can be prevented (Coleman et al., 2012), complications and deaths can be averted resulting in reduced overall outbreak costs (Ghebrehewet et al., 2016). Complications such as encephalitis can require up to 8 months of rehabilitation (Suijkerbuijk et al., 2015) and the average cost of a measles case complicated by encephalitis is $\$ 50,500, \$ 70,059$ and $\$ 132,487$ or $\$ 6535, \$ 9173$ and $\$ 9544$ for febrile convulsion complications in the Netherlands, UK and Canada, respectively. This is far above \$276, \$307 and $\$ 254$ spent on average per case for uncomplicated measles in these same countries (Carabin et al., 2002).

The high disparity in outbreak response cost per case in the included studies could be explained by the corresponding difference in the contacts per case ratios which has been shown to have the highest impact on CT costs during sensitivity analysis (Canadian Agency for Drugs and Technologies in Health, 2015). However, this rising CT costs per case can be mitigated if CT results in a short outbreak duration. This is because the extent of public health response is directly proportional to not just the number of cases plus contacts but to also the length of the outbreak with the mean costs of small outbreaks ranging from $\$ 2685$ to $\$ 22,000$, from $\$ 58,000$ to $\$ 146,000$ for medium outbreaks, and from $\$ 551,000$ to $\$ 985,000$ for large outbreaks (Ortega-Sanchez et al., 2014). This cost per case variance in the selected studies may also be as a result of case count differences $(\$ 18,000 /$ case for 22 cases, and $\$ 439 /$ case for 12,427 cases). This finding would concur with the evidence that public health response costs are inversely proportional to the number of cases in an outbreak and are a function of the number of contacts traced (Ghebrehewet et al., 2016).

In this systematic review, labour costs dominated health sector-related outbreak costs while household-related costs were driven by the opportunity costs of workdays lost by cases/caregivers. Evidence on measles outbreak and associated economic costs show that the health authorities bear most of the costs owing mainly to intensified surveillance, media communications, case registration workload and use of experts while productivity losses are partly attributed to childcare (Suijkerbuijk et al., 2015). In addition, there is substantial evidence that illness-related expenses are overwhelming and driving poverty among households in LMICs (McIntyre et al., 2006).

From the included studies in this systematic review, the overall outbreak cost is influenced by the range of costs considered in cost analysis and the extent to which each outbreak response intervention is applied. It has been shown that the size of an outbreak affects the type and intensity of response and thus resources required (Canadian Agency for Drugs and Technologies in Health, 2015). Evidence from the health sector perspective shows that outbreak response is not costeffective ( $€ 524,735 / \mathrm{QALY}$ compared to the threshold of $€ 35,500 / \mathrm{QALY})$ for few preventable measles cases. However, this finding is only generalisable to countries that have achieved measles elimination (Ramsay et al., 2019) and therefore suggest that CT could be cost-effective in LMICs where the prospects of large measles outbreaks are high, and elimination has not been achieved.

In these unprecedented times of the coronavirus disease2019 pandemic, a new highly infectious, droplet and contact borne viral disease, the WHO's provisional guidelines acknowledged the central role played by $\mathrm{CT}$ in outbreak investigations when implemented systematically and on time to break rapidly growing transmission chains. Across the different settings in which transmission can take place, the importance of interviewing cases to identify contacts is highlighted. Also, establishing lists of individuals (where possible) who have shared spaces with cases or warning/informing any potential contacts was emphasised (WHO, 2020). These methods of identifying contacts were common across the studies included in this systematic review and reiterates the relevance of timely and organised CT efforts as the world prepares for a potential second wave of the coronavirus disease-2019 pandemic.

\section{Limitations}

Only four included studies evaluated the economic costs of CT and because CT was jointly costed with other outbreak response activities, it was difficult to determine what proportion of these costs were attributed to CT alone. Future studies should consider disaggregating data so that the direct and indirect costs of $\mathrm{CT}$ can be measured separately from the costs of other outbreak investigation activities. An update of this systematic review will also be needed as journal articles 
addressing the research question could not be found in the searched databases.

In addition, the findings of this systematic review are prone to bias. It is possible that only studies with significant results were published and thus selected for this review, introducing selection bias. Language bias could arise from restricting the inclusion of papers to only those published in English. There is also a potential for publication bias in this systematic review as grey literature was not reviewed.

\section{Conclusion}

To the best of our knowledge, this systematic review is the first to be conducted on CT methods and its associated costs in LMICs during measles outbreaks. Results indicate that there is a paucity of CT data as a standalone and comparative active surveillance approach in LMICs. Results also suggest that CT by recall and shared space identification are common during outbreaks in LMICs. However, CT based on contacts recalled by cases may not be very comprehensive compared to that by shared space identification. Using a measles case as a focal point, CT can adopt a backward or forward perspective or a combination of the two by using the exposure and infective periods of the case to determine who the case was exposed to, and who may have been exposed to the case. Thus, CT can be viewed as a continuum of 'from whom' (source/backward) 'to whom' (secondary cases or forward). Evidence suggests that the cost of CT per measles case is high, and is dominated by labour expenses from the health sector perspective and productivity losses from the household perspective. The overall outbreak costs can be low if CT is timely and leads to a reduced outbreak size and/or duration. This will reduce the economic burden of measles and drive LMICs towards measles elimination. In this coronavirus disease-2019 era, it is important for governments to allocate sufficient CT resources to maximise the benefits of early and organised CT implementation should a second wave of the pandemic occur. This COVID-19 pandemic could increase the competition for the already endangered measles campaign resources with an associated increase in the risks of larger outbreaks in the future.

Abbreviations CASP, Critical Appraisal Skills Programme; CHEERS, Consolidated Health Economic Evaluation Reporting Standards; CT, Contact tracing; $€$, Euros; LMICs, Low- and middle-income countries; PICO, Population intervention comparator outcome; PRISMA, Preferred reporting items for systematic reviews and meta-analyses; PROSPERO, Prospective register of systematic reviews; QALY, Quality adjusted life year; QHES, Quality of health economic studies; STROBE, Strengthening the reporting of observational studies in epidemiology; \$/USD, United States Dollars; UK, United Kingdom; USA, United States of America; WHO, World Health Organization.
Supplementary Information The online version contains supplementary material available at https://doi.org/10.1007/s10389-021-01590-2.

Authors' contribution ELM developed the main concepts for this systematic review, conducted preliminary database search, article screening, selection and analysis, and discussed the findings with major inputs from ML who performed the second independent article screening/selection and provided feedback critical to the overall conceptualisation and implementation of this review. As the third reviewer, JCH resolved article selection disagreements between ELM and ML. ELM wrote the first draft of the manuscript in collaboration with ML and JCH who edited and provided remarks for its improvement.

Data availability All data generated or analysed during this study are included in this published article and its online source files deposited in the figshare repository.

Code availability Not applicable.

\section{Declarations}

Ethics approval The study was registered with the Academic Ethics Committee for Bangor University and was granted exemption from requiring ethics approval.

Consent to participate Not applicable.

Consent for publication Not applicable.

Competing interests The authors have no relevant financial or nonfinancial interests to disclose.

\section{References}

Aggarwal R, Ranganathan P (2019) Study designs: part 2 - descriptive studies. Perspect Clin Res 10:34-36. https://doi.org/10.4103/picr. PICR_154_18

Abad CL, Safdar N (2015) The reemergence of measles. Curr Infect Dis Rep 17:51. https://doi.org/10.1007/s11908-015-0506-5

Beard FH, Franklin LJ, Donohue SD et al (2011) Contact tracing of inflight measles exposures: lessons from an outbreak investigation and case series, Australia, 2010. W Pacific Surveill Response J 2:e1-e1. https://doi.org/10.5365/wpsar.2011.2.2.010

Begum L, Chow Y, Falola A, Meltzer M, Shah N (2017) A measles outbreak in the London borough of Hammersmith and Fulham, September-November 2016. Eur J Pub Health 27:147. https://doi. org/10.1093/eurpub/ckx187.375

Canadian Agency for Drugs and Technologies in Health (2015) Costs of contact tracing activities aimed at reducing the transmission of measles in Canada. https://www.ncbi.nlm.nih.gov/books/NBK304885/. Accessed 1 March 2019

Carabin H, Edmunds WJ, Kou U, van den Hof S, Nguyen VH (2002) The average cost of measles cases and adverse events following vaccination in industrialised countries. BMC Public Health 2:22. https:// doi.org/10.1186/1471-2458-2-22

CASP (2018) CASP checklists - CASP - Critical Appraisal Skills Programme. https://casp-uk.net/casp-tools-checklists/. Accessed 20 April 2019

Centre for Reviews and Dissemination University of York (2009) Systematic reviews: CRD's guidance for undertaking reviews in 
health care. https://www.york.ac.uk/crd/guidance/. Accessed 19 April 2019

Chen SY, Anderson S, Kutty PK et al (2011) Health care-associated measles outbreak in the United States after an importation: challenges and economic impact. J Infect Dis 203:1517-1525. https:// doi.org/10.1093/infdis/jir115

Ching PK, Zapanta MJ, de Los Reyes VC, Tayag E, Magpantay R (2016) Investigation of a measles outbreak in cordillera, northern Philippines, 2013. W Pacific Surveill Response J 7:1-5. https:// doi.org/10.5365/WPSAR.2015.6.4.007

Cisse B, Aaby P, Simondon F, Samb B, Soumare M, Whittle H (1999) Role of schools in the transmission of measles in rural Senegal: implications for measles control in developing countries. Am J Epidemiol 149:295-301. https://doi.org/10.1093/oxfordjournals. aje.a009811

Coleman MS, Garbat-Welch L, Burke H et al (2012) Direct costs of a single case of refugee-imported measles in Kentucky. Vaccine 30: 317-321. https://doi.org/10.1016/J.VACCINE.2011.10.091

Dabbagh A, Laws RL, Steulet C et al (2018) Progress towards regional measles elimination - worldwide, 2000-2017. Wkly Epidemiol Rec 93:649-660 https://apps.who.int/iris/bitstream/handle/10665/ 276217/WER9348.pdf. Accessed 10 June 2019

Dhillon RS, Srikrishna D (2018) When is contact tracing not enough to stop an outbreak? Lancet Infect Dis 18:1302-1303. https://doi.org/ 10.1016/S1473-3099(18)30469-9

Mbivnjo EL, Lynch M, Huws JC (2021) Supplementary material for the article, "measles outbreak investigation process in low- and middleincome countries: a systematic review of the methods and costs of contact tracing". Figshare Dataset. https://doi.org/10.6084/m9. figshare. 14247431

Garenne M, Aaby P (1990) Pattern of exposure and mealses mortality in Senegal. J Infect Dis 132:1088-1094. https://doi.org/10.1093/infdis/ 161.6.1088

Ghebrehewet S, Thorrington D, Farmer S et al (2016) The economic cost of measles: healthcare, public health and societal costs of the 201213 outbreak in Merseyside, UK. Vaccine 34:1823-1831. https://doi. org/10.1016/J.VACCINE.2016.02.029

Goodson JL, Perry RT, Mach O et al (2010) Measles outbreak in Tanzania, 2006-2007. Vaccine 28:5979-5985. https://doi.org/10. 1016/j.vaccine.2010.06.110

Hales CM, Johnson E, Helgenberger L et al (2016) Measles outbreak associated with low vaccine effectiveness among adults in Pohnpei state, Federated States of Micronesia, 2014. Open Forum Infect Dis 3:1-7. https://doi.org/10.1093/ofid/ofw064

Hinman AR (2018) Measles and rubella eradication. Vaccine 36:1-3. https://doi.org/10.1016/J.VACCINE.2017.11.062

Husereau D, Drummond M, Petrou S et al (2013) Consolidated health economic evaluation reporting standards (CHEERS) statement. $\mathrm{Br}$ Med J (Clinical Research Edition) 346:f1049. https://doi.org/10. 1136/bmj.f1049

Jin Y, Ma H, Zhang L et al (2011) Measles outbreak on a college campus transmitted through internet cafés. J Infect Dis 204:471-475. https:// doi.org/10.1093/infdis/jir069

Jones J, Klein R, Popescu S et al (2015) Lack of measles transmission to susceptible contacts from a health care worker with probable secondary vaccine failure - Maricopa County, Arizona, 2015. Morb Mortal Wkly Rep 64:832-833 http://www.ncbi.nlm.nih.gov/ pubmed/26247437. Accessed 29 September 2019

Lemos DRQ, Franco AR, Roriz MLFdeS et al (2017) Measles epidemic in Brazil in the post-elimination period: coordinated response and containment strategies. Vaccine 35:1721-1728. https://doi.org/10. 1016/j.vaccine.2017.02.023

Ma C, Yan S, Su Q et al (2016) Measles transmission among adults with spread to children during an outbreak: implications for measles elimination in China, 2014. Vaccine 34:6539-6544. https://doi.org/10. 1016/j.vaccine.2016.02.051
Ma R, Lu L, Suo L et al (2017) An expensive adult measles outbreak and response in office buildings during the era of accelerated measles elimination, Beijing, China. Vaccine 35:1117-1123. https://doi.org/ 10.1016/j.vaccine.2017.01.021

Marin M, Nguyen HQ, Langidrik JR et al (2006) Measles transmission and vaccine effectiveness during a large outbreak on a densely populated island: implications for vaccination policy. Clin Infect Dis 42: 315-319. https://doi.org/10.1086/498902

McIntyre D, Thiede M, Dahlgren G, Whitehead M (2006) What are the economic consequences for households of illness and of paying for health care in low- and middle-income country contexts? Soc Sci Med 62:858-865. https://doi.org/10.1016/J.SOCSCIMED.2005.07. 001

Measles and Rubella Initiative (2018) 2017 in Numbers. https://monzo. com/blog/2018/01/05/2017-in-numbers/. Accessed 10 April 2019

Moher D, Liberati A, Tetzlaff J, Altman DG, Group TP (2009) Preferred reporting items for systematic reviews and meta-analyses: the PRISMA statement. PLoS Med 6:e1000097. https://doi.org/10. 1371/journal.pmed.1000097

Monten C, Veldeman L, Verhaeghe N, Lievens Y (2017) A systematic review of health economic evaluation in adjuvant breast radiotherapy: quality counted by numbers. Radiother Oncol : J Eur Soc Therapeutic Radiol Oncol 125:186-192. https://doi.org/10.1016/j. radonc.2017.08.034

Moss WJ (2017) Measles. Lancet 390:2490-2502. https://doi.org/10. 1016/S0140-6736(17)31463-0

Mupere E, Karamagi C, Zirembuzi G et al (2006) Measles vaccination effectiveness among children under 5 years of age in Kampala, Uganda. Vaccine 24:4111-4115. https://doi.org/10.1016/j.vaccine. 2006.02.038

Njau J, Janta D, Stanescu A et al (2019) Assessment of economic burden of concurrent measles and rubella outbreaks, Romania, 2011-2012. Emerg Infect Dis 25:1101-1109. https://doi.org/10.3201/eid2506. 180339

Ofman JJ, Sullivan SD, Neumann PJ et al (2003) Examining the value and quality of health economic analyses: implications of utilizing the QHES. J Manag Care Pharm 9:53-61. https://doi.org/10.18553/ jmcp.2003.9.1.53

Orenstein WA, Hinman A, Nkowane B, Olive JM, Reingold A (2018) Measles and rubella global strategic plan 2012-2020 midterm review. Vaccine 36:A1-A34. https://doi.org/10.1016/J.VACCINE. 2017.09.026

Ortega-Sanchez IR, Vijayaraghavan M, Barskey AE, Wallace GS (2014) The economic burden of sixteen measles outbreaks on United States public health departments in 2011. Vaccine 32:1311-1317. https:// doi.org/10.1016/J.VACCINE.2013.10.012

Piatti-Fünfkirchen M, Lindelow M, Yoo K (2018) What are governments spending on health in east and southern africa? Health Syst Reform 4:284-299. https://doi.org/10.1080/23288604.2018.1510287

Pike J, Tippins A, Nyaku M, Eckert M, Helgenberger L, Underwood JM (2017) Cost of a measles outbreak in a remote island economy: 2014 Federated States of Micronesia measles outbreak. Vaccine 35:59055911. https://doi.org/10.1016/j.vaccine.2017.08.075

Quiroga R, Barrezueta O, Venczel L et al (2003) Interruption of indigenous measles transmission in Bolivia since October 2000. J Infect Dis 187:S121-S126. https://doi.org/10.1086/368051

Ramsay LC, Crowcroft NS, Thomas S et al (2019) Cost-effectiveness of measles control during elimination in Ontario, Canada, 2015. Euro Surveillance : Euro Comm Dis Bull 24:1800370. https://doi.org/10. 2807/1560-7917.ES.2019.24.11.1800370

Rathi P, Narendra V, Sathiya V et al (2017) Measles outbreak in the adolescent population - matter of concern? J Clin Diagn Res 11: LC20-LC23. https://doi.org/10.7860/JCDR/2017/28619.10488

Sarmiento H, Cobo OB, Morice A, Zapata R, Benitez MV, CastilloSolórzano C (2011) Measles outbreak in Venezuela: a new 
challenge to postelimination surveillance and control? J Infect Dis 204:675-682. https://doi.org/10.1093/infdis/jir444

Siani A (2019) Measles outbreaks in Italy: a paradigm of the reemergence of vaccine-preventable diseases in developed countries. Prev Med 121:99-104. https://doi.org/10.1016/j.ypmed.2019.02. 011

Smith K (2018) Preventing, identifying and managing measles outbreaks. Nurs Times 144:18-19 https://www.nursingtimes.net/clinicalarchive/infection-control/preventing-identifying-and-managingmeasles-outbreaks-28-08-2018/.

Sniadack DH, Crowcroft NS, Durrheim DN, Rota PA (2017) Roadmap to elimination- standard measles and rubella surveillance. Wkly Epidemiol Rec 92:97-116 https://apps.who.int/iris/bitstream/ handle/10665/254652/WER9209-10.pdf?sequence=1.

Strebel PM, Cochi SL, Hoekstra E et al (2011) A world without measles. J Infect Dis 204:S1-S3. https://doi.org/10.1093/infdis/jir111

Suijkerbuijk AWM, Woudenberg T, Hahné SJM et al (2015) Economic costs of measles outbreak in the Netherlands, 2013-2014. Emerg Infect Dis 21:2067-2069. https://doi.org/10.3201/eid2111.150410

von Elm E, Altman DG, Egger M et al (2007) The strengthening the reporting of observational studies in epidemiology (STROBE) statement: guidelines for reporting observational studies. Lancet (London, England) 370:1453-1457. https://doi.org/10.1016/ S0140-6736(07)61602-X

Wallace AS, Masresha BG, Grant G et al (2014) Evaluation of economic costs of a measles outbreak and outbreak response activities in Keffa zone, Ethiopia. Vaccine 32:4505-4514. https://doi.org/10.1016/j. vaccine.2014.06.035

Whittle HC, Aaby P, Samb B, Jensen H, Bennett J, Simondon F (1999) Effect of subclinical infection on maintaining immunity against measles in vaccinated children in West Africa. Lancet 353:98102. https://doi.org/10.1016/S0140-6736(98)02364-2
WHO (2012) Global Measles and rubella strategic plan 2012-2020. http://www.who.int/about/licensing/copyright_form/en/index.html. Accessed 18 April 2019

WHO (2015) Emergency guideline: Implementation and management of contact tracing for Ebola virus disease. https://apps.who.int/iris/ bitstream/handle/10665/185258/WHO_EVD_Guidance_Contact 15.1 eng.pdf?sequence $=1$. Accessed 25 February 2019

WHO (2017) Measles vaccines: WHO position paper, April 2017. Wkly Epidemiol Rec 92:205-228 https://apps.who.int/iris/bitstream/ handle/10665/255149/WER9217.pdf?sequence $=1$. Accessed 10 July 2019

WHO (2018) Guidance for evaluating progress towards elimination of measles and rubella. Wkly Epidemiol Rec 93:541-552 https://apps. who.int/iris/bitstream/handle/10665/275392/WER9341.pdf. Accessed 10 July 2019

WHO (2019) New measles surveillance data for 2019. https://www.who. int/immunization/newsroom/measles-data-2019/en/. Accessed 18 April 2019

WHO (2020) Contact tracing in the context of COVID-19. https://www. who.int/publications-detail/contact-tracing-in-the-context-of-covid19. Accessed 30 August 2020

WHO Regional Committee for Africa (2017) Progress towards measles elimination in the African Region by 2020. http://apps.who.int/ immunization_monitoring/globalsummary/timeseries/ tscoveragebcg.html. Accessed 18 April 2019

Zhang DL, Pan JR, Xie SY et al (2015) A hospital-associated measles outbreak among individuals not targeted for vaccination in eastern China, 2014. Vaccine 33:4100-4104. https://doi.org/10.1016/j. vaccine.2015.06.066

Publisher's note Springer Nature remains neutral with regard to jurisdictional claims in published maps and institutional affiliations. 